\title{
Haftung einer Wirtschaftsprüfungsgesellschaft für einen fehlerhaften Prüfbericht in einem Wertpapierprospekt
}

\author{
Besprechung von BGH Urt. vom 24.4.2014 - III ZR 156/13*
}

Prof. Dr. Dres. h.c. Werner F. Ebke, LL.M. (UC Berkeley), Heidelberg***

Die Entscheidung des BGH behandelt die zivilrechtliche Haftung einer Wirtschaftsprüfungsgesellschaft gegenüber Wertpapiererwerbern für die Erteilung eines Prüfberichts über eine Gewinnprognose in einem Wertpapierprospekt gemäß VO (EG) Nr.809/2004, die sich später als fehlerhaft herausstellt. Die Wirtschaftsprüfungsgesellschaft gehörte nicht zu den Prospektverantwortlichen (Prospektherausgeber; Prospektveranlasser) im Sinne der damals geltenden $\iint 44 f f$ Börs G a.F. i. V.m. $\iint 8 f$, 13 VerkProspG a.F. Der BGH entschied, dass diese Vorschriften den Rückgriff auf das allgemeine Zivilrecht nicht sperren, und verurteilte die Beklagte auf der Grundlage des Rechtsinstituts des Vertrages mit Schutzwirkung für Dritte, dem Wertpapiererwerber den Verlust des für den Erwerb der Anteile an der inzwischen insolventen Emittentin aufgewandten Betrages zu ersetzen. Die Entscheidung gibt Anlass zu zablreichen Fragen aus dogmatischer und wirtschaftlicher Sicht.

The Supreme Court's decision deals with the civil liability of an accounting firm towards persons who acquired shares of an unlisted corporation in reliance upon a prospectus which contained, inter alia, a profit forecast for the year 2007 which was false and misleading. The accounting firm had prepared a report in accordance with Regulation (EC) Nr. 809/2004, stating that in its opinion the forecast had been "properly compiled on the basis stated and that the basis of accounting used for the profit forecast or estimate is consistent with the accounting policies of the issuer". The Securities Exchange Act (SEA - Börsengesetz) in force at the relevant time provided an express civil liability for false or misleading statements in a prospectus but imposed this liability only upon persons who had initiated or signed the prospectus. The Supreme Court held, however, that courts may resort to general contract law principles to bold accountants liable to purchasers of securities even if the accountants were not liable under the express prospectus liability provision of the SEA. The decision gives rise to numerous doctrinal and economic issues.

BGB $\int \ 199$ Abs. 1 Nr. 2, 328, 675; WpPG $\$ 7$

1. Zur Haftung einer Wirtschaftsprüfungsgesellschaft, die in einem Wertpapierprospekt ein fehlerhaftes Testat betreffend die Prüfung der Gewinnprognosen nach $\$ 7$ des Wertpapierprospektgesetzes i. V. m. Art. 3 und Anhang I Nr. 13.2 der VO (EG) Nr. 809/2004 abgegeben hat, gegenüber einem Kapital-

* U.a. veröffentlicht in DB 2014, 1126 = NJW 2014, $2345=$ NZG 2014, $741=$ WM 2014, $935=$ ZIP 2014, 972.

** Werner F. Ebke, Dr. iur., Dr. rer. pol. h.c., Dr. iur. h.c., LL.M. (UC Berkeley), Universitätsprofessor für Bürgerliches Recht, deutsches europäisches und Internationales Unternehmensrecht sowie Direktor des Instituts für deutsches und europäisches Gesellschafts- und Wirtschaftsrecht, Ruprecht-Karls-Universität Heidelberg; Attorneyat-Law (New York). 
anleger nach den Grundsätzen des Vertrags mit Schutzwirkung zugunsten Dritter.

2. Liegt der haftungsauslösende Fehler der Wirtschaftsprüfungsgesellschaft in einer falschen Rechtsanwendung, beginnt die regelmäßige Verjährungsfrist nach $\$ 199$ Abs. 1 Nr. 2 BGB nicht bereits mit dem Schluss des Jahres, in dem der Geschädigte Kenntnis von der Rechtsanwendung als solcher erlangt hat; vielmehr muss der Geschädigte Kenntnis oder grob fahrlässig Unkenntnis davon haben, dass die Rechtsanwendung fehlerhaft gewesen ist (im Anschluss an BGH, Urteil vom 6. Februar 2014 - IX ZR 245/12, NJW-RR 2014, 993 = WM 2014, 575).

\section{Sachverbalt}

Die Kl. macht aus abgetretenem Recht ihres Ehemanns (im Folgenden: Zedent) Schadensersatzansprüche gegen die beklagte Wirtschaftsprüfungsgesellschaft wegen eines unrichtigen Prüfberichts geltend. Im Jahre 2007 legte die T-AG zwecks Ausgabe von Namensaktien einen Wertpapierprospekt auf. Dieser enthielt auf den S. 53-59 Planrechnungen für die Jahre 2007-2011, aus denen die für diese Geschäftsjahre zu Grunde gelegten Gewinnprognosen und Gewinnschätzungen ersichtlich waren. Im März 2007 hatte die T-AG die Bekl. beauftragt, die Rechnungslegungsgrundlagen der Gewinnprognosen und Gewinnschätzungen gemäß der EG-Verordnung Nr. 809/2004 in Verbindung mit dem Wertpapierprospektgesetz zu prüfen. In dem Prüfungsvertrag zwischen der T-AG und der Bekl. vom 21.3.2007 wurde die Geltung der „Allgemeinen Auftragsbedingungen für Wirtschaftsprüfer und Wirtschaftsprüfungsgesellschaften“ (AAB) vom 1.1.2002 vereinbart. Der in dem Prospekt auf S. 60-62 abgedruckte Prüfbericht der Bekl. vom 25. 4. 2007 endete mit der zusammenfassenden Feststellung, dass die Gewinnprognosen und Gewinnschätzungen des Emittenten in Übereinstimmung mit den angegebenen Grundlagen ordnungsgemäß aufgestellt worden seien und dass diese Grundlagen im Einklang mit den Rechnungslegungsstrategien der Gesellschaft stünden. Im Juli 2007 erwarb der Zedent eine Beteiligung an der T-AG zum Nennwert von $9.000 €$. Der Zedent hat die entsprechenden Aktien nie erhalten. Die Kapitalerhöhung, imZuge derer die Aktien emittiert werden sollten, ist nicht in das Handelsregister eingetragen worden. Die T-AG ist insolvent.

Das LG Duisburg gab der Klage auf Schadensersatz im Wesentlichen statt. ${ }^{1}$ Das OLG Düsseldorf hat die Berufung der Bekl. zurückgewiesen. ${ }^{2}$ Die zugelassene Revision hatte keinen Erfolg.

1 LG Duisburg, Urt. v. 11.11.2011 - 10 O 65/11, BeckRS 2014, 09272.

2 OLG Düsseldorf, Urt. v. 5. 4. 2013 - I-17 U 185/11, BeckRS 2014, 17539. 


\section{Bedeutung der Entscheidung}

Die vorstehende Entscheidung des III.Zivilsenats des BGH ist ein weiterer Baustein einer Rechtsprechung, die aus Gründen des Kapitalanlegerschutzes auf die Verschärfung der zivilrechtlichen Verantwortlichkeit von Wirtschaftsprüfern und Wirtschaftsprüfungsgesellschaften gegenüber Dritten gerichtet ist und in zahlreichen Punkten über die vertrag(sähn)liche Dritthaftung des Wirtschaftsprüfers in Pflichtprüfungsfällen gemäß $₫ 323$ Abs. 1 Satz 3 HGB hinausgeht ${ }^{3}$. Zu diesem Zweck setzt der Senat in casu auf das Rechtsinstitut des Vertrages mit Schutzwirkung für Dritte, dessen Anwendung dogmatische Zweifel aufwirft und wirtschaftlich unerwünschte Folgen haben kann. Die Chance, ein rechtlich stimmiges und wirtschaftlich tragfähiges Konzept für die Haftung des Wirtschaftsprüfers für fehlerhafte Beiträge zu Wertpapierprospekten jenseits der gesetzlich geregelten Prospekthaftung zu schaffen, bot sich dem Senat in casu nicht. Das ist umso bedauerlicher, als sich Untergerichte gerade mit der Haftung von Wirtschaftsprüfern gegenüber Kapitalanlegern wegen unrichtiger oder unvollständiger Angaben in Wertpapierprospekten außerhalb der gesetzlich geregelten Prospekthaftung schwer tun ${ }^{4}$. Das zu besprechende Urteil hat weitreichende Auswirkungen über den entschiedenen Fall hinaus, auch wenn die streitgegenständlichen Vorschriften der $\$ \mathbb{S} 44 \mathrm{ff}$ BörsG a.F. i. V.m. $\int \mathbb{S} 8$ f, 13 VerkProspG a.F. mit Wirkung vom 1.6.2012

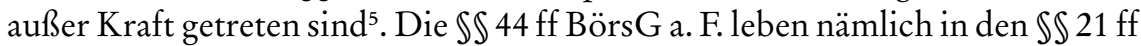

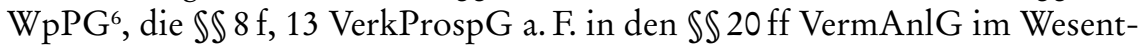
lichen fort ${ }^{7}$.

\section{Der Fall}

In dem der hier zu besprechenden Entscheidung des III.Zivilsenats des BGH zugrunde liegenden Fall geht es um den Prüfbericht einer unabhängigen Wirtschaftsprüfungsgesellschaft über Gewinnprognosen in einem Wertpapierprospekt. Ein solcher Prüfbericht ist nach $\$ 7 \mathrm{WpPG}$ in Verbindung mit Art. 3

$3 \mathrm{Zu}$ Einzelheiten der Haftung des gesetzlichen Jahresabschlussprüfers siehe statt aller Eвке, Münchener Komm. z. HGB, Bd. 4, 3. Aufl., 2013, \$323 Rdn. 20 ff (Haftung gegenüber der Mandantin) und Rdn. $85 \mathrm{ff}$ (Haftung gegenüber Dritten).

4 Ein erschreckendes Beispiel aus jüngerer Zeit ist LG Hamburg, Urt. v. 12. 6. 2013-309 O 425/08, http: \\openjur.de/u/635428.html (n.rk.).

5 Art. 7 des Gesetzes zur Novellierung des Finanzanlagenvermittler- und Vermögensanlagenrechts vom 6.12.2011, BGBl. I, S. 2481.

$6 \mathrm{Zu}$ Einzelheiten der Haftung nach $\$ \$ 21 \mathrm{ff}$ WpPG und den Unterschieden zu $\$ \int 44$ BörsG a.F. siehe Buck-HeEb, Kapitalmarktrecht, 7.Aufl., 2014, S.69-71; Grunewald/ SchlitT, Einführung in das Kapitalmarktrecht, 3. Aufl., 2014, S. 236-245.

$7 \mathrm{Zu}$ Einzelheiten der Haftung nach $\$ \mathbb{S} 20 \mathrm{ff}$ VermAnlG und den Unterschieden zu $\$ 13$ VerkProspG a. F. siehe BucK-НеEв, aаO (Fn. 6), S. 72-76. 
Abs. 2 Satz 1 und Ziffer 13.2 des Anhangs I der seit dem 1. Juli 2005 geltenden VO (EG) Nr. 809/20048 in Wertpapierprospekten im Sinne der Richtlinie 2003/71/EG9 erforderlich, wenn sich der Emittent (Anbieter) dazu entschließt, in den Prospekt eine Gewinnprognose ${ }^{10}$ oder eine Gewinnschätzung ${ }^{11}$ aufzunehmen. Der Prospekt muss dann einen Bericht enthalten, „der von unabhängigen Buchprüfern oder Abschlussprüfern erstellt wurde und in dem festgestellt wird, dass die Prognose oder die Schätzung nach Meinung der unabhängigen Buchprüfer oder Abschlussprüfer auf der angegebenen Grundlage ordnungsgemäß erstellt wurde und dass die Rechnungslegungsgrundlage, die für die Gewinnprognose oder -schätzung verwendet wurde, mit den Rechnungslegungsstrategien des Emittenten kohärent ist" (Ziffer 13.2 des Anhangs I zu der VO (EG) Nr. 809/2004).

Das OLG Düsseldorf ist in seinem Berufungsurteil davon ausgegangen, dass zwischen den Parteien unstreitig sei, dass der Prüfbericht jedenfalls bezüglich der Planrechnung für das Jahr 2007 unrichtig war. ${ }^{12}$ Entgegen den in dem Prospekt vom 25. April 2007 abgedruckten Planrechnungen für die Jahre 2007 bis 2011, die für das Jahr 2007 Ausschüttungen in Höhe von 278.000,00 Euro prognostizierten, durften gemäß $\$ 269$ Satz 2 HGB a.F. zumindest in dem ersten Jahr des Planungszeitraums keine Ausschüttungen an die Aktionäre der T-AG vorgenommen werden. $\$ 269$ Satz 2 HGB a. F. bestimmte nämlich, dass ein Unternehmen, das von seinem Aktivierungswahlrecht nach $\$ 269$ Satz 1 HGB Gebrauch macht, Aufwendungen für die Ingangsetzung des Geschäftsbetriebs und dessen Erweiterung (in casu 2.835.000,00 Euro) in der Bilanz auszuweisen, ${ }^{13}$ Gewinne nur ausschütten darf, „wenn die nach der

8 Verordnung (EG) Nr. 809/2004 der Kommission vom 29. April 2004 zur Umsetzung der Richtlinie 2003/71/EG des Europäischen Parlaments und des Rates betreffend die in Prospekten enthaltenen Informationen sowie das Format, die Aufnahme von Informationen mittels Verweis und die Veröffentlichung solcher Prospekte und die Verbreitung von Werbung, ABlEU L 149 v. 30.4.2004, S. 1.

9 Richtlinie 2003/71/EG des Europäischen Parlaments und des Rates vom 4. November 2003 betreffend den Prospekt, der beim öffentlichen Angebot von Wertpapieren oder bei deren Zulassung zum Handel zu veröffentlichen ist und zur Änderung der Richtlinie 2001/34/EG, ABl.EU L 345 v. 31.12.2003, S. 64.

10 Art. 2 Ziffer 10 der VO (EG) Nr. 809/2004 bezeichnet „Gewinnprognose“ als „einen Text, in dem ausdrücklich oder implizit eine Zahl oder eine Mindest- bzw. Höchstzahl für die wahrscheinliche Höhe der Gewinne und Verluste im laufenden Geschäftsjahr und/oder in den folgenden Geschäftsjahren genannt wird, oder der Daten enthält, aufgrund deren die Berechnung einer solchen Zahl für die künftigen Gewinne oder Verluste möglich ist, selbst wenn keine bestimmte Zahl genannt wird und das Wort, Gewinn“ nicht erscheint".

11 „Gewinnschätzung“ ist nach Art. 2 Ziffer 11 der VO (EG) Nr. 809/2004 „eine Gewinnprognose für ein abgelaufenes Geschäftsjahr, für das die Ergebnisse noch nicht veröffentlicht wurden“.

12 OLG Düsseldorf BeckRS 2014, 17539.

$13 \mathrm{Zu}$ Einzelheiten des $\$ 269$ HGB a. F. siehe zuletzt KreIde, Selbstgeschaffene immate- 
Ausschüttung verbleibenden jederzeit auflösbaren Gewinnrücklagen zuzüglich eines Gewinnvortrags und abzüglich eines Verlustvortrags dem angesetzten Betrag mindestens entsprechen“. Die Ausschüttungssperre bezweckte, dass das bilanzielle Vermögen des Unternehmens aus Gründen des Gläubigerschutzes nicht durch überhöhte Ausschüttungen unter die Summe des gezeichneten Kapitals und der Rücklagen sinkt. ${ }^{14}$ Die zusammenfassende Feststellung der Bekl. in ihrem „Bericht über die Prüfung des Prospektes über Aktien“ auf Seite 62 des Prospekts, dass die darin enthaltenen Gewinnprognosen und Gewinnschätzungen des Emittenten nach ihrer Auffassung in Übereinstimmung mit den angegebenen Grundlagen ordnungsgemäß aufgestellt worden seien und diese Grundlagen mit den Rechnungslegungsstrategien der Gesellschaft in Einklang stünden, war daher unrichtig. Die Unrichtigkeit beruhte auf einer fehlerhaften Rechtsanwendung. Gegen die diesbezüglichen Feststellungen des Berufungsgerichts wandte sich die Bekl. in ihrer Revision nicht ${ }^{15}$.

\section{Börsengesetzliche oder kapitalmarktrechtliche Haftung}

Ob die Bekl. der Kl. deswegen nach börsengesetzlichen oder kapitalmarktrechtlichen Vorschriften auf Schadensersatz haftet, haben das LG Duisburg ${ }^{16}$ und das OLG Düsseldorf ${ }^{17}$ - soweit ersichtlich - nicht in Erwägung gezogen. Der III.Zivilsenat des BGH gibt in einem Nebensatz zu erkennen, dass eine börsengesetzliche Prospekthaftung der Bekl. nicht in Betracht komme, weil die Bekl. „nicht zu den Prospektverantwortlichen (Prospektherausgeber; Prospektveranlasser) im Sinne der gesetzlichen Prospekthaftung .... gehöre“18.

\section{Adressaten der börsengesetzlichen Prospekthaftung}

Die gesetzliche Prospekthaftung nach den bis zum 1.6.2012 geltenden $\$ \llbracket 44 \mathrm{ff}$

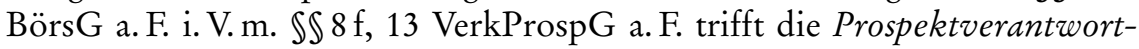
lichen, also diejenigen Personen, die für den Prospekt die Verantwortung übernommen haben ( $\$ 44$ Abs. 1 Satz 1 Nr. 1 BörsG a. F.), und die Prospektveranlasser, also diejenigen Personen, von denen der Erlass des Prospekts ausgeht ( $\$ 44$ Abs. 1 Satz 1 Nr. 2 BörsG a. F.).Zu den Prospektverantwortlichen

rielle Vermögensgegenstände im Recht der Rechnungslegung junger Technologieunternehmen, 2014, S. 168-173.

14 Reiner, Münchener Komm. z. HGB, Bd. 4, 2. Aufl., 2008, \$269 Rdn. 12.

15 BGH NZG 2014, 741, 743 (Rdn. 23).

16 LG Duisburg BeckRS 2014, 09272.

17 OLG Düsseldorf BeckRS 2014, 17539.

18 BGH NZG 2014, 741, 743 (Rdn. 16). 
zählen die Unterzeichner des Prospekts ${ }^{19}$ und die im Prospekt als Verantwortliche aufgeführten Personen ${ }^{20}$. Wenn - was zu vermuten steht - die Bekl. den Prospekt nicht unterzeichnet hat und sie in dem Prospekt nicht als „Verantwortliche" für die Richtigkeit und Vollständigkeit der Prospektangaben aufgeführt war, gehört sie in der Tat nicht zu dem Kreis der Prospektverantwortlichen und haftet daher auch nicht nach $₫ 44$ Abs. 1 Satz 1 Nr. 1 BörsG a.F. i. V.m. $\int \mathbb{\int} 8$ f, 13 VerkProspG a.F. Nach $\$ 44$ Abs. 1 Satz 1 Nr. 2 BörsG a.F. haften ferner diejenigen Personen, von denen der Prospekt ausgeht (Prospektveranlasser). Mit dieser Vorschrift versucht der Gesetzgeber, Haftungslücken zu schließen, die sich ergeben können, wenn die Emission von Personen initiiert und betrieben wird, die entweder ein eigenes wirtschaftliches Interesse daran haben oder auf die Erstellung des Prospekts maßgeblichen Einfluss genommen haben, aber keine Prospektverantwortung übernehmen ${ }^{21}$. Typische Beispiele für Prospektveranlasser sind Großaktionäre oder Muttergesellschaften, insbesondere wenn sie im Rahmen der Platzierung eigene Aktien anbieten ${ }^{22}$. Als Prospektveranlasser kommen aber auch Mitglieder des Vorstands oder des Aufsichtsrats des Emittenten in Betracht, wenn sie die Prospekterstellung wesentlich steuern und ein über die Organstellung hinausgehendes erhebliches wirtschaftliches Eigeninteresse an der Emission haben ${ }^{23}$. Nach dem von den Untergerichten in casu mitgeteilten Sachverhalt ist nicht anzunehmen, dass die Bekl. Prospektveranlasser im Sinne des $\mathbb{} 44$ Abs. 1 Satz 1 Nr. 2 BörsG a.F. war.

\section{Teilverantwortung}

Bei der börsengesetzlichen Prospekthaftung handelt es sich nach herrschender Meinung um eine Verantwortung für den gesamten Prospekt, nicht für einzelne Teile oder Angaben des Prospekts. Das folgt bereits aus dem Wortlaut des $₫ 44$ Abs. 1 Börs G a. F., der sprachlich von einer Haftung für den gesamten Prospekt ausgeht. Experten, insbesondere Abschlussprüfer, Sachverständige und Rechtsanwälte, die bei der Herstellung des Prospekts Angaben oder Materialien für den Prospekt geliefert oder an dessen Erstellung mitgewirkt haben, haften deshalb im Regelfall nicht nach den börsengesetzlichen Prospekthaftungsvorschriften des $\$ 44$ Abs. 1 Satz 1 Nr. 2 BörsG a. F., auch nicht für die

19 Unterzeichner des Prospekts sind der Emittent der Wertpapiere (vgl. $\ 5$ Abs. 3 Satz 2 WpPG) und, wenn auf Grund des Prospekts Wertpapiere zum Handel an einem organisierten Markt zugelassen werden, der antragstellende Emissionsbegleiter (vgl. $\ 5$ Abs. 3 Satz 2 WpPG). Der antragstellende Emissionsbegleiter ist auch schon nach $\ 5$ Abs. 4 Satz 2 WpPG im Prospekt als Verantwortlicher aufgeführt.

20 Langenbucher, Aktien- und Kapitalmarktrecht, 2. Aufl., 2011, S. 276.

21 Langenbucher, aaO (Fn. 20), S. 277.

22 KRÄMER, in: Krieger/Schneider, Handbuch Managerhaftung, 2. Aufl., 2010, S. 814, 827.

23 Vgl. BGHZ 123, 106, 110 (betr. Vorstandsmitglied). 
Teile des Prospekts, die sie geliefert haben, weil sie nicht die Verantwortung für den gesamten Prospekt übernehmen ${ }^{24}$. Dem kann nicht mit Erfolg entgegengehalten werden, die Angaben der Bekl. seien untrennbar mit dem Gesamtinhalt des Prospekts verwoben, so dass sich ihre Verantwortung gar nicht auf einen isoliert abgrenzbaren Prospektteil beschränken lasse. Der in Rede stehende unrichtige Teil des Prüfberichts beschränkt sich im Wesentlichen auf die Gewinnprognosen für die Jahre 2007-2011. Dieser Teil des Prospekts ist eingrenzbar und eigenständig, da die Angaben zu den Gewinnprognosen aus der Rechnungslegung abgeleitet werden. Demgegenüber enthalten zahlreiche andere Angaben in dem Prospekt gemäß den Anhängen zu der VO (EG) Nr. 809/2004 unternehmensbezogene Angaben und zukunftsgerichtete unternehmenspolitische Aussagen und Wertungen, die sich nicht aus der Rechnungslegung ableiten lassen. Der Grundsatz, dass es für die börsengesetzliche Prospekthaftung auf die Verantwortung für den gesamten Prospekt ankommt, steht entgegen der Ansicht von Gro $\beta^{25}$ nicht im Widerspruch zu der VO (EG) Nr. 809/2004. Ziffern 1.1 und 1.2 des Anhangs III der ProspektVO erwähnen zwar die Verantwortung für bestimmte Abschnitte des Prospekts. Das bedeutet aber nur, dass nach den Vorstellungen des Verordnungsgebers Personen die Verantwortung für bestimmte Teile oder Angaben des Prospekts übernehmen können, die in dem Prospekt angegeben sind. Die Verordnung schließt danach die Übernahme einer Teilverantwortlichkeit nicht aus, sofern die Urheber der Teile oder Angaben in dem Prospekt die börsengesetzliche Prospektverantwortung für einzelne Teile oder Angaben tatsächlich übernommen haben. Eine Vorgabe dahingehend, dass eine Teilverantwortung in jedem Fall anzunehmen ist, ist der VO (EG) Nr. 809/2004 einschließlich ihrer Anhänge nicht $\mathrm{zu}$ entnehmen ${ }^{26}$. Die Übernahme einer Teilverantwortung entspricht regelmäßig - wie auch in dem vorliegenden Fall - nicht dem Willen des Wirtschaftsprüfers. Die Bekl. hatte, soweit ersichtlich, auch kein eigenes wirtschaftliches Interesse an der Emission an sich ${ }^{27}$. Das Honorarinteresse reicht nach zutreffender Ansicht für das notwendige wirtschaftliche Eigeninteresse jedenfalls nicht aus ${ }^{28}$. Im Ergebnis scheidet eine Haftung der Bekl. auf Grund der $\$ \$ 44 \mathrm{ff}$ BörsG a. F. i. V.m. $\int \mathbb{S} 8$ f, 13 VerkProspG a. F. mithin in der Tat aus.

24 Grunewald/Schlitt, aaO (Fn.6), S. 240 m.w. N.; Langenbucher, aaO (Fn. 20), S. 277 m. w. N.; Schwark, Börsengesetz, 2. Aufl., 1994, $\$ \int 45$, 46 Rdn. 7; vgl. Kumpan, in: Baumbach/Норт, Handelsgesetzbuch, 36. Aufl., 2014, WpPG $\$ 21$ Rdn. 4 („Wirtschaftsprüfer haften für die von ihnen verantworteten Teile nach [ $\$ 21$ Abs. 1 Satz 1 Nr. 1 WpPG]").

25 Gross, Kapitalmarktrecht, 4. Aufl., 2009, $\int \$ 44,45$ BörsG Rdn. 36.

26 Grunewald/SchlitT, aaO (Fn. 6), S. 240 m. w. N.

27 Siehe dazu LG Frankfurt a. M. WM 1998, 1181, 1183.

28 Langenbucher, aaO (Fn. 20), S. 278 unter Hinweis auf Assmann, AG 2004, 435 ff; Grunewald/Schlitt, aaO (Fn. 6), S. 240. 


\section{Zivilrechtliche Haftung}

Welche Folgen hat diese Feststellung für die von dem III. Zivilsenat angenommene Haftung der Bekl. nach den Grundsätzen des Vertrages mit Schutzwirkung für Dritte? Konkret: Sperren die $\$ \mathbb{S} 44 \mathrm{ff}$ BörsG a. F. i. V.m. $\int \mathbb{S} 8 \mathrm{f}, 13$ VerkProspG a.F. die Annahme eines Vertrages mit Schutzwirkung für Dritte, wie die Bekl. unter Hinweis auf die parallele Diskussion zu $\$ 323$ HGB meint?

\section{Sperrwirkung?}

Der III. Zivilsenat des BGH hat in seiner Entscheidung vom 2. 4. 1998, die eine gesetzliche Jahresabschlussprüfung nach $\mathbb{S} 316 \mathrm{ff}$ HGB betraf, eine „Sperrwirkung“ des $₫ 323$ Abs. 1 Satz 3 HGB verneint: „Wenn $\ 323$ Abs. 1 Satz 3 HGB eine gesetzliche Haftung (nur) gegenüber der Kapitalgesellschaft und dem verbundenen Unternehmen regelt“, bedeute dies nicht, dass „damit eine vertragliche Haftung des Abschlußprüfers gegenüber Dritten nach Maßgabe der von der Rechtsprechung entwickelten Grundsätze zur Dritthaftung Sachkundiger von vornherein ausgeschlossen wäre“29. „Eine derartige Sperrwirkung gegenüber der Möglichkeit einer interessengerechten, auch dem Grundsatz der Privatautonomie Rechnung tragenden Gestaltung der Haftungsbedingungen“ sei der Vorschrift „in dieser Allgemeinheit nicht zu entnehmen“30. Eine Dritthaftung, die „wesentlich“ darauf beruhe, dass „es Sache der Vertragsparteien ist zu bestimmen, gegenüber welchen Personen eine Schutzpflicht begründet werden soll“, werde „von dem unmittelbaren Anwendungsbereich des $₫ 323$ Abs. 1 HGB nicht berührt" und bedeute auch nicht „eine Mißachtung einer in dieser Vorschrift zum Ausdruck gekommenen Grundentscheidung des Gesetzgebers für eine begrenzte Dritthaftung von Wirtschaftsprüfern“31. Einen „so weitgehenden Zweck“ verfolge $\$ 323$ Abs. 1 Satz 3 HGB nicht ${ }^{32}$. Mehr als ein obiter dictum sind diese Ausführungen freilich nicht. Denn das die Haftung auslösende Verhalten des Abschlussprüfers bestand nicht in einer fehlerhaften Abschlussprüfung oder der Erteilung eines unrichtigen Bestätigungsvermerks bzw. der unberechtigten Versagung eines Bestätigungsvermerks über die durchgeführte Pflichtprüfung ( $\$ 322 \mathrm{HGB}$ ), sondern in einem Fehler im Zusammenhang mit der Ankündigung eines bestimmten Testats vor Abschluss der Prüfung und Erteilung des Bestätigungsvermerks. Ein solches Verhalten fällt jedenfalls nach herrschender und zutreffender Meinung von vornherein nicht in den Anwendungsbereich des $₫ 323$

29 BGH JZ 1998, 1013 mit Aufsatz Eвке, JZ 1998, 991.

30 BGH JZ 1998, 1013, 1014.

31 BGH JZ 1998, 1013, 1014.

32 BGH JZ 1998, 1013, 1014. 
Abs. $1 \mathrm{HGB}^{33}$. Die Frage einer „Sperrwirkung“ wurde am Ende auch offen gelassen. Der Senat wies die Sache nämlich zwecks weiterer tatrichterlicher Feststellungen an das Berufungsgericht (OLG Hamm) zurück, das Gelegenheit erhielt, im Rahmen der erneuten Würdigung darauf einzugehen, ob sich ein Anspruch des Kl. auf Auskunftsvertrag oder Delikt stützen lasse ${ }^{34}$.

Hoffnung schöpfte die Bekl. in casu aber möglicherweise aus einigen jüngeren Entscheidungen des III. Zivilsenats des BGH, in denen der Senat seine grundsätzliche Ablehnung einer „Sperrwirkung“ des $₫ 323$ Abs. 1 Satz 3 HGB zwar bestätigt, in einigen zentralen Punkten aber wichtige Klarstellungen vorgenommen hat. So hebt der Senat in seinem Urteil vom 6. 4.2006 ${ }^{35}$, das ebenfalls einen Pflichtprüfungsfall betrifft, hervor, dass „die gesetzgeberische Intention, das Haftungsrisiko des Abschlussprüfers angemessen zu begrenzen, auch im Rahmen der vertraglichen Dritthaftung zu beachten sei und die Einbeziehung einer unbekannten Vielzahl von Gläubigern, Gesellschaftern oder Anteilserwerbern in den Schutzbereich des Prüfauftrags dieser Tendenz zu-

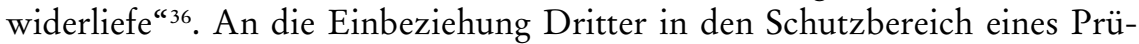
fungsauftrags seien daher „strenge Anforderungen“ zu stellen $^{37}$. Die von dem Senat als „restriktiv verstandene Anwendung von Grundsätzen der vertraglichen Dritthaftung im Bereich der Pflichtprüfung" sei auch im Hinblick auf das Gesetzgebungsverfahren zu dem am 1.5.1998 in Kraft getretenen Gesetz zur Kontrolle und Transparenz im Unternehmensbereich vom 27.4.1998 38 „geboten“39. In seinem Urteil vom 11.11.2008 hat der III.Zivilsenat des BGH seine Position bekräftigt, dass $\$ 323$ HGB zwar nicht „von Rechts wegen aus[schließt], dass für den Abschlussprüfer auf vertraglicher Grundlage auch eine Schutzpflicht gegenüber dritten Personen begründet werden kann“; an die „Annahme einer vertraglichen Einbeziehung eines Dritten in den Schutzbereich“ des Prüfungsvertrages seien aber „strenge Anforderungen zu stellen“" ${ }^{40}$. Für die Annahme einer Schutzwirkung genüge es nicht, „dass ein Dritter die von Sachkunde geprägte Stellungnahme des Prüfers für diesen erkennbar zur Grundlage einer Entscheidung mit wirtschaftlichen Folgen machen möchte“41. Der Senat betont, es „wäre ein Verstoß gegen die gesetzliche Wertung des $\$ 323$ Abs. 1 Satz 3 HGB, wenn man ... annehmen wollte,

33 Eвке, aаO (Fn. 3), \323 HGB Rdn. 22-25 m. w. N.; offenlassend BGH JZ 1998, 1013, 1014.

34 Die Sache wurde schließlich durch Vergleich erledigt: Eвке, WPK-Mitt. 1999, 114, 114 Fn. 5.

35 BGHZ 167, 155.

36 BGHZ 167, 155, 163.

37 BGHZ 167, 155, 163-164.

38 BGBl. I S. 786. Zu Einzelheiten des Gesetzes und seine Auswirkungen auf $\$ 323$ Abs. 1 Satz 3 HGB siehe EBKe, aaO (Fn. 3), \323 HGB Rdn. 154-155.

39 BGHZ 167, 155, 163.

40 BGH BeckRS 2008, 24194 (betr. Dritthaftung im Rahmen von Pflichtprüfungen).

41 BGH BeckRS 2008, 24194. 
der Pflichtprüfer übernehme ohne besonderen Anlass und ohne Gegenleistung - gewissermaßen in doppelter Hinsicht konkludent - sowohl die Begründung als auch die mögliche Vervielfältigung seiner Haftung “42.

\section{Einschlägig?}

Die von der Revision angeführten Senatsurteile vom 6.4.2006 und vom 2. 4.1998 hält der III. Zivilsenat des BGH in casu allerdings nicht für „einschlägig "43. Einen entscheidenden Unterschied sieht der Senat darin, dass es sich bei Jahresabschlussprüfungen nach $\$ \mathbb{S} 316 \mathrm{ff} H G B$ um gesetzlich vorgeschriebene Prüfungen handele, wohingegen die Prüfung von Gewinnprognosen und Gewinnschätzungen in Wertpapierprospekten davon abhänge, „ob sich der Emittent, um sein Angebot für die Kunden besonders attraktiv zu machen, entschließt, Gewinnerwartungen in den Prospekt aufzunehmen“"44. „Erst und nur dann“, so der Senat, „sollen diese zum Schutz der Anleger durch einen Wirtschaftsprüfer kontrolliert und das Ergebnis der Prüfung den Anlegern über die Veröffentlichung im Prospekt zugänglich gemacht werden“ “45. Diese Ausführungen nehmen indes eine Differenzierung vor, wo es keine wesentlichen Unterschiede gibt. Schließlich beruht die gesetzlich vorgeschriebene Prüfung von Jahresabschlüssen ebenfalls auf einer privatautonomen Entscheidung, nämlich der Wahl, in der Rechtsform einer prüfungspflichtigen Gesellschaft geschäftlich tätig zu werden ${ }^{46}$. Dass in dem einen Fall die Prüfung handelsrechtlich, in dem anderen börsengesetzlich bzw. kapitalmarktrechtlich vorgeschrieben ist, kann insoweit keinen Unterschied machen, zumal beide Prüfungspflichten auf europarechtlichen Vorgaben beruhen.

Der Senat begründet seine Ansicht, dass die erwähnten Urteile nicht einschlägig seien, ferner damit, dass sich die streitgegenständlichen Regelungen „deutlich vom Regelungsgefüge der $\mathbb{S} 316 \mathrm{ff} \mathrm{HGB}$ “ unterscheiden, weil die börsengesetzlich vorgeschriebene Prüfung der Gewinnprognosen und Gewinnschätzungen „auch und gerade den Schutz der konkreten Anleger“ bezwecke; Bestätigungsvermerken von Pflichtprüfern komme dagegen die Bedeutung zu, „allgemein Dritten einen Einblick in die wirtschaftliche Situation des publizitätspflichtigen Unternehmens zu gewähren und ihnen - sei es als

42 BGH BeckRS 2008, 24194. In seinem Urteil vom 7. 5. 2009 hat der Senat die Bedeutung der konkret-individuellen Ermittlung des Drittschutzes im Zusammenhang mit einer Sonderprüfung noch einmal bekräftigt: BGHZ 181, 12.

43 BGH NZG 2014, 741, 743 (Rdn. 21).

44 BGH NZG 2014, 741, 743 (Rdn. 21).

45 BGH NZG 2014, 741, 743 (Rdn. 21).

$46 \mathrm{Zu}$ den Gesellschaften, die gesetzlich verpflichtet sind, ihre Jahresabschlüsse durch einen unabhängigen Wirtschaftsprüfer prüfen zu lassen, siehe Евке, aаO (Fn. 3), $\$ 316$ HGB Rdn. 2-9. 
künftigen Kunden beziehungsweise Gläubigern, sei es als an einer Beteiligung Interessierten - für ihr beabsichtigtes Engagement eine Beurteilungsgrundlage zu geben “47. Warum der ausgemachte Unterschied - hier der Bestätigungsvermerk über die Prüfung der vergangenheitsbezogenen Darstellung der Vermögens-, Finanz- und Ertragslage des geprüften Unternehmens, dort der Prüfbericht über den Inhalt zukunftsorientierter Planrechnungen mit Gewinnprognosen des Emittenten - gegen die Übertragbarkeit von Überlegungen zu der behaupteten „Sperrwirkung“ einer sonderprivatrechtlichen Haftungsnorm des Handelsrechts auf börsen- bzw. kapitalmarktrechtliche Haftungsregeln sprechen soll, erschließt sich allerdings nicht ohne Weiteres. Entgegen der Ansicht des III.Zivilsenats des BGH spricht auch nicht gegen die Annahme einer „Sperrwirkung“, dass nach $\ 47$ Abs. 2 BörsG a. F. „neben den gesetzlichen Prospekthaftungsansprüchen weitergehende vertragliche Ansprüche unberührt bleiben " ${ }^{48}$. Eine entsprechende Bestimmung findet sich in $\$ 323$ HGB nicht; trotzdem nimmt der Senat an, dass neben dieser Norm weitergehende vertrag(sähn)liche und deliktsrechtliche Ansprüche Dritter bestehen können. $\$ 47 \mathrm{Abs.} 2$ BörsG a. F. spräche nur dann für die Annahme, dass $\int \mathbb{S} 44 \mathrm{ff}$ BörsG a.F. i. V.m. $\int \mathbb{S} 8$ f, 13 VerkProspG a. F. den Rückgriff auf die Grundsätze der vertraglichen Dritthaftung nicht sperren, wenn $\ 47$ Abs. 2 BörsG a. F. nicht nur weitergehende (z. B. über die Haftungsausschlüsse nach $\$ 45$ BörsG a. F. hinausgehende) Ansprüche „auf Grund von Verträgen oder vorsätzlichen oder grob fahrlässigen unerlaubten Handlungen“ gegen die in \44 Abs. 1 BörsG a. F. genannten Adressaten der Prospekthaftung unberührt lassen will ${ }^{49}$, sondern darüber hinaus Wertpapiererwerbern vertragliche bzw. deliktsrechtliche Ansprüche wegen unrichtiger oder unvollständiger Prospektangaben auch gegenüber Personen eröffnen soll, die nicht zu dem Kreis der Prospektverantwortlichen bzw. Prospektveranlasser im Sinne von $\mathbb{} 44$ Abs. 1 Satz 1 Nr. 1 und 2 BörsG a. F. gehören. Das ist aber nicht anzunehmen. Nach Wortlaut, systematischer Stellung sowie Sinn und Zweck des $₫ 47$ Abs. 2 BörsG a. F. sprechen die besseren Gründe dafür, dass diese Bestimmung nur weitergehende Ansprüche gegen die Prospektverantwortlichen bzw. Prospektveranlasser im Blick hat und keine Ansprüche von Wertpapiererwerbern gegen Personen begründen will, die nicht Adressaten der börsengesetzlichen Prospekthaftung $\operatorname{sind}^{50}$.

47 BGH NZG 2014, 741, 743 (Rdn. 21).

48 BGH NZG 2014, 741, 743 (Rdn. 21). \47 Abs. 2 BörsG a. F. lautet: „Weitergehende Ansprüche, die nach den Vorschriften des bürgerlichen Rechtes auf Grund von Verträgen oder vorsätzlichen oder grob fahrlässigen unerlaubten Handlungen erhoben werden können, bleiben unberührt“.

$49 \mathrm{Zu}$ den Änderungen auf Grund von $₫ 25$ Abs. 2 WpPG siehe unten bei Fn. 63.

50 In diesem Sinne auch Gross, aaO (Fn. 25), $\$ 47$ BörsG Rdn. 2 („\$47 Abs. 2 bestimmt wie auch schon in der Fassung vor dem Dritten Finanzmarktförderungsgesetz zunächst, dass vertragliche und nebenvertragliche Ansprüche gegen einen Prospektverantwort- 


\section{Regelungsgefüge}

Die von dem III. Zivilsenat des BGH angesprochenen unterschiedlichen Regelungsgefüge der streitgegenständlichen Vorschriften und des $₫ 323$ Abs. 1 Satz 3 HGB sind allerdings aus einem anderen Grund bedeutsam. Wenn man mit dem Senat davon ausgeht, dass $₫ 323$ Abs. 1 Satz 3 HGB den Rückgriff auf die Grundsätze der vertraglichen Dritthaftung nicht sperrt und eine solche

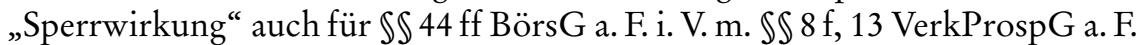
ablehnt, stellt sich die Frage, ob sich dann zumindest die „restriktiv verstandene Anwendung von Grundsätzen der vertraglichen Dritthaftung im Bereich der Pflichtprüfung " ${ }^{51}$ auf die vertragliche Dritthaftung für einen unrichtigen Prüfbericht über Gewinnprognosen nach $\$ \mathbb{S} 44 \mathrm{ff}$ BörsG a. F. i. V. m. $\$ \mathbb{S} 8 \mathrm{f}, 13$ VerkProspG a. F. übertragen lässt. Der Senat führt in diesem Zusammenhang aus: Ungeachtet der ,auf Publizität und Vertrauensbildung angelegten Funktion" der Abschlussprüfung habe der Gesetzgeber die Verantwortlichkeit des Abschlussprüfers für eine Pflichtprüfung in $\$ 323$ Abs. 1 Satz 3 HGB auf Ansprüche der Kapitalgesellschaft und verbundener Unternehmen beschränkt und diese bei Fahrlässigkeit zudem der Höhe nach begrenzt ( $\$ 323$ Abs. 2 HGB $)^{52}$. Gläubigern sowie Aktionären hafte der Prüfer nach dieser Bestimmung nicht ${ }^{53}$. Vor dem Hintergrund dieser „gesetzgeberischen Wertentscheidung" habe der Senat „auch die Möglichkeit einer Haftung nach den Grundsätzen des Vertrages mit Schutzwirkung zu Gunsten Dritter eingeschränkt " ${ }^{\text {" }}$. In der Tat ist die Haftung des Pflichtprüfers gegenüber prüfungsvertragsfremden Dritten nach der jüngeren Rechtsprechung des III. Zivilsenats des $\mathrm{BGH}^{55}$ und zahlreicher Obergerichten ${ }^{56}$ grundsätzlich auf Fälle vorsätzlicher Pflichtverletzungen beschränkt ( $\mathbb{S} 823$ Abs. 1, 823 Abs. 2 i. V.m. Schutzgesetz, 826 $\mathrm{BGB})^{57}$, sofern die Parteien des Prüfungsvertrages den gesetzlich beschränkten Schutzbereich des Prüfungsvertrages nicht ausnabmsweise auf einen oder mehrere Dritte erstreckt haben ${ }^{58}$ oder es durch Kontaktaufnahme des Ab-

lichen durch $\int \mathbb{S} 44 \mathrm{ff}$ nicht ausgeschlossen werden“) (Hervorhebung vom Verf.); ebenso Buck-Heeb, aaO (Fn. 6), S. 72.

51 BGHZ 167, 155, 163.

52 BGH NZG 2014, 741, 743 (Rdn. 21).

53 BGH NZG 2014, 741, 743 (Rdn. 21).

54 BGH NZG 2014, 741, 743 (Rdn. 21). Die Einschränkung gilt nach Ansicht des Senats auch für Abschlussprüfungen, die nach den für die Pflichtprüfung maßgeblichen \\316ff HGB freiwillig vorgenommen werden: BGH WM 2006, 423 (betr. Dritthaftung für gesetzlich nicht vorgeschriebene Abschlussprüfung einer $\mathrm{GmbH}$ gegenüber zukünftigen Genussrechtserwerbern).

55 Евке, aаO (Fn. 3), \323 HGB Rdn. 136, 140, 143 m. w. N.

56 Eвке, aаO (Fn. 3), \323 HGB Rdn. 144 m. w. N.

$57 \mathrm{Zu}$ der deliktsrechtlichen (Dritt-)Haftung des Pflichtprüfers siehe EвкE, aaO (Fn. 3), \323 HGB Rdn. 92-113.

58 Eвкe, aaO (Fn. 3), \323 HGB Rdn. 136, 164. 
schlussprüfers mit dem Dritten (z. B. durch Zusendung des testierten Jahresabschlusses) zu einem eigenständigen Vertragsverhältnis (z. B. Auskunftsvertrag gemäß $\$ 675$ Abs. 2 BGB) zwischen dem Prüfer und dem Dritten kommt ${ }^{59}$. Diese Risikoallokation in dem Verhältnis von Abschlussprüfer, Mandantin und Dritten unterscheidet sich sachlich und methodisch von der Risikoverteilung nach den streitgegenständlichen Regelungen.

Die $\int \mathbb{S} 44 \mathrm{ff}$ BörsG a.F. i. V.m. $\mathbb{S} 8 \mathrm{f}, 13$ VerkProspG a. F. verteilen allfällige Haftungsrisiken für unrichtige oder unvollständige Prospektangaben in dem Verhältnis von Emittent, Wirtschaftsprüfer und Wertpapiererwerber, indem sie zum Beispiel den Kreis der Haftpflichtigen auf Prospektverantwortliche und Prospektveranlasser begrenzen ( $\$ 44$ Abs. 1 Satz 1 Nr. 1 und 2 BörsG a. F.), die Ersatzberechtigung an enge Fristen seit der Erstveröffentlichung knüpfen ( $\$ 44$ Abs. 1 Satz 1 BörsG a. F.), nur Erwerbsgeschäfte erfassen, die im Inland abgeschlossen worden sind oder denen ganz oder teilweise im Inland erbrachte Wertpapierdienstleistungen zugrunde liegen ( $\$ 44 \mathrm{Abs.} 3$ BörsG a. F.) und zahlreiche Haftungsausschlüsse vorsehen ( $\$ 45$ BörsG a. F.), wobei zwischen individueller Entlastung mangels Verschuldens ( $\$ 45$ Abs. 1 BörsG a.F.) und allgemeinen Entlastungen etwa durch den Nachweis fehlender haftungsbegründender ( $\$ 45$ Abs. $2 \mathrm{Nr} .1$ BörsG a. F.) oder haftungsausfüllender Kausalität ( $\ 45$ Abs. 2 Nr. 2 BörsG a. F.) unterschieden wird ${ }^{60}$. Hinzu kommt, dass die Rechtsfolge nicht voller Schadensersatz, sondern „Rückabwicklung“ des Erwerbsgeschäfts ist, wobei $\$ 44$ BörsG a. F. danach differenziert, ob der Anspruchssteller noch Inhaber der Wertpapiere ist oder nicht $(\$ 44 \mathrm{Abs} .1$ Satz 1 , Abs. 2 BörsG a. F.). Ähnlich wie nach $\ 323$ Abs. 4 HGB kann die Ersatzpflicht nach $₫ 44$ Börs $\mathrm{a}$ a. F. vertraglich weder beschränkt noch erlassen werden $(\$ 47$ Abs. 1 BörsG a. F.).

Auf Grund der Haftungsausschlüsse, vor allem aber wegen der Vorschrift des $\$ 47$ Abs. 2 BörsG a. F., dass neben einem Anspruch von Wertpapiererwerbern aus $\mathbb{\int} 44$ ff BörsG a. F. i. V. m. $\int \mathbb{S} 8 \mathrm{f}, 13$ VerkProspG a. F. nur deliktsrechtliche Ansprüche in Betracht kommen, die auf einem vorsätzlichen oder grob fahrlässigen Verhalten des Prospektverantwortlichen bzw. Prospektveranlassers beruhen, kann sich die merkwürdige Situation ergeben, dass Personen, die nicht Adressaten der Prospekthaftung nach $₫ 44$ Abs. 1 Satz 1 Nr. 1 oder 2 BörsG a. F. sind (wie etwa Wirtschaftsprüfer, Rechtsanwälte oder andere Experten, die bei der Erstellung des Prospekts mitgewirkt oder Angaben zu dem Prospekt beigesteuert haben, ohne Verantwortung für den Prospekt zu übernehmen), gegenüber Wertpapiererwerbern schärfer haften als diejenigen, die für den unrichtigen Prospekt (ganz oder teilweise) Verantwortung übernom-

59 In der Praxis sind derartige Ausnahmen in Pflichtprüfungsfällen auf Grund standesrechtlicher Regelungen höchst selten. Siehe Ebke, aaO (Fn. 3), \$323 HGB Rdn.124, 127.

60 Buck-Неeb, aaO (Fn. 6), S. 70; JäGer, Das Prospekthaftungsstatut, 2007, S. 53-64. 
men haben oder von denen der Erlass des unrichtigen Prospekts ausgegangen ist. Denn wenn das Gericht beispielsweise einen Wirtschaftsprüfer in den Schutzbereich des Vertrages zwischen ihm und dem Emittenten über die Prüfung von Gewinnprognosen einbezieht, haftet der Wirtschaftsprüfer Wertpapiererwerbern schon für leichte Fahrlässigkeit, wohingegen die Haftung der Prospektverantwortlichen und Prospektveranlasser nach $\mathbb{\$} \$ 44$ Abs. 1 Satz 1 Nr. 1 und 2, 45 Abs. 2, 47 Abs. 2 BörsG a.F. auf Vorsatz und grobe Fahrlässigkeit beschränkt ist, sofern sie mit den Wertpapiererwerbern nicht ausnahmsweise weitergehende Ansprüche vertraglich vereinbart haben (vgl. \47 Abs. 2 BörsG a. F.).

In casu spielte diese Überlegung zwar keine Rolle, weil die Bekl. nach den Feststellungen des OLG Düsseldorf „zumindest grob fahrlässig “ gehandelt hatte ${ }^{61}$. In Fällen leichter Fahrlässigkeit der Experten stellt sich aber die Frage, ob deren Haftung nach den Grundsätzen der vertraglichen Dritthaftung nicht entsprechend der börsengesetzlichen Prospekthaftung auf Vorsatz und grobe Fahrlässigkeit beschränkt werden müsste, falls die Experten keine Prospektverantwortung übernommen haben; andernfalls würde man einen offenen Wer-

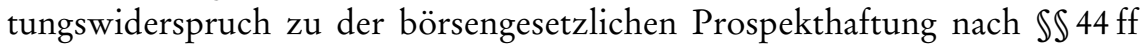
BörsG a. F. i. V. m. $\int \mathbb{S} 8$ f, 13 VerkProspG a. F. in Kauf nehmen. Diese Frage stellt sich vor allem im Hinblick auf die "Altfälle“ nach $\mathbb{\int} 44 \mathrm{ff}$ BörsG a. F. i. V.m. $\$ \int 8 \mathrm{f}, 13$ VerkProspG a. F. Denn anders als nach $\$ 47$ Abs. 2 BörsG a. F. besteht gemäß $\ 25$ Abs. 2 WpPG keine Beschränkung auf Vorsatz oder grobe Fahrlässigkeit; vielmehr kommen danach deliktsrechtliche Ansprüche auch bei lediglich leicht fahrlässigem Verhalten der Prospektverantwortlichen bzw. Prospektveranlasser in Betracht, so dass ein Wertungswiderspruch jedenfalls bezüglich des Verschuldens nicht besteht. Dennoch existiert zwischen den Grundsätzen der vertraglichen Dritthaftung und $\$ 25$ Abs. 2 WpPG ein Regelungsgefälle. Denn wegen der hinlänglich bekannten „Enge“ des deutschen Rechts im Hinblick auf den deliktsrechtlichen Schutz des Vermögens an sich ${ }^{62}$ hat die Haftungsausweitung auf Grund von $\$ 25$ Abs. 2 WpPG in der Praxis nur eine geringe Bedeutung ${ }^{63}$, wohingegen den Grundsätzen der vertraglichen Dritthaftung eine entsprechende Enge des Schutzes fremden Vermögens unbekannt ist. Zur Vermeidung schwerwiegender Wertungswidersprüche müssen die Gerichte bei ihrer Suche nach „einer interessengerechten, auch den Grundsatz der Privatautonomie Rechnung tragenden Gestaltung der Haftungsbedingungen" ${ }^{\text {"64 }}$ außerhalb der gesetzlich geregelten Prospekthaftung m.E. - genauso wie in den jüngeren Entscheidungen des III. Zivilsenats des BGH zu Pflicht-

61 OLG Düsseldorf BeckRS 2014, 17539. Deshalb kam in casu eine Haftung der Bekl. für den unrichtigen Prüfbericht nach $\$ 826$ BGB nicht in Betracht.

62 Евке, aаO (Fn. 3), $\$ 323$ HGB Rdn. 92 ff.

63 Leuering, NJW 2012, 1905, 1906.

64 BGH JZ 1998, 1013, 1014. 
prüfungsfällen ${ }^{65}$ - mit Augenmaß vorgehen und eine „praktische Konkordanz“ (Konrad Hesse) herstellen zwischen der gesetzlichen Prospekthaftung der Prospektverantwortlichen bzw. Prospektveranlasser und der vertraglichen Dritthaftung von Wirtschaftsprüfern und anderen Experten, die Angaben oder Materialien für den Prospekt geliefert oder an dessen Erstellung mitgewirkt haben, ohne Verantwortung für den Prospekt übernommen zu haben ${ }^{66}$. Die kollidierenden, zu schützenden Interessen der Beteiligten müssen in der Problemlösung einander so zugeordnet werden, dass jedes von ihnen Wirklichkeit gewinnt und beide zur optimalen Wirksamkeit gelangen.

\section{Vertrag mit Schutzwirkung für Dritte}

Das gilt umso mehr, als das Institut des Vertrages mit Schutzwirkung für Dritte eine schillernde Rechtsfigur ist, deren ständige Ausweitung auch und gerade im Bereich der Haftung des Wirtschaftsprüfers ${ }^{67}$ sowohl das OLG Düsseldorf ${ }^{68}$ als auch der III. Zivilsenat des $\mathrm{BGH}^{69}$ anschaulich nachgezeichnet haben.

\section{Voraussetzungen}

Der III.Zivilsenat des BGH sieht in casu die Voraussetzungen eines Schutzwirkung für Dritte entfaltenden Vertrages als gegeben $\mathrm{an}^{70}$. Über das Vorliegen der klassischen vier Voraussetzungen ${ }^{71}$ lässt sich in dem vorliegenden Fall trefflich streiten. Besonders hervorhebenswert ist der Hinweis des Senats auf die dogmatische Grundlage des von der Rechtsprechung entwickelten Rechtsinstituts des Vertrages mit Schutzwirkung für Dritte: Dieses beruhe, so betont der Senat, ,auf einer maßgeblich durch das Prinzip von Treu und Glauben $(\mathbb{2} 242$ BGB) geprägten ergänzenden Vertragsauslegung $(\mathbb{\$} 157$ BGB)“72. Damit setzt sich der Senat von Autoren ab, die den Drittschutz als Gewohnheitsrecht, Ausfluss eines allgemeinen, auf das Vertrauensprinzip oder schlicht auf den Grundsatz von Treu und Glauben ( $\$ 242$ BGB) gegrün-

65 Siehe oben Fn. 35-42.

66 Ein solcher Ansatz würde u. a. bedeuten, dass Anlageinteressenten, die im Vertrauen auf den Prospekt von dem Erwerb von Wertpapieren abgesehen haben, keinen Schadensersatzanspruch gegen den Prüfer haben wegen späterer höherer Gewinne oder eines nicht antizipierten Wertzuwachses der Wertpapiere.

67 Eвке, aаO (Fn. 3), \323 HGB Rdn. $132 \mathrm{ff}$.

68 OLG Düsseldorf BeckRS 2014, 17539.

69 BGH NZG 2014, 741, 741-742 (Rdn. 10 ff) („Ausgangspunkt“, „Weiterentwicklung“).

70 BGH NZG 2014, 741, 743 (Rdn. 12-24).

71 Siehe dazu statt vieler Eвke, aaO (Fn. 3), \$323 HGB Rdn. 133-134 m. w. N.

72 BGH NZG 2014, 741, 741 (Rdn. 9). 
deten, von dem Willen der Beteiligten unabhängigen Schuldverhältnisses begreifen und daher zum Beispiel auf das Erfordernis der Erkennbarkeit der sog. Leistungsnähe und des Drittschutzinteresses verzichten ${ }^{73}$. Ob „ein rechtsgeschäftlicher Wille zur Einbeziehung" bestehe, habe - so betont der Senat - der Richter nach allgemeinen Auslegungsregeln zu ermitteln ${ }^{74}$. Da eine ausdrückliche Einigung über die Einbeziehung der Wertpapiererwerber in den Schutzbereich des Prüfungsvertrages zwischen dem Emittenten und dem Prüfer regelmäßig fehlen wird, liegt es nahe, nach einer allfälligen „stillschweigenden" Einigung der Parteien über die Externalisierung der vertraglichen Schutzpflichten zu fragen. Die Annahme einer solchen „stillschweigenden“ Einigung beruht aber oft nicht auf dem freien rechtsgeschäftlichen Willen der Parteien und auch nicht auf einem (geschriebenen) Rechtssatz, sondern letztlich auf dem Gedanken der Risikobeherrschung und Zurechnung und gerät sehr schnell in die Nähe der Fiktion. Die von dem Senat herangezogene ergänzende Vertragsauslegung ( $\$ 157 \mathrm{BGB})$ ist ebenfalls nicht unproblematisch, muss man doch darauf abstellen, was die Parteien bei einer angemessenen Abwägung ihrer Interessen nach Treu und Glauben ( $\$ 242$ BGB) als redliche Vertragspartner vereinbart hätten, wenn sie den von ihnen nicht geregelten Fall bedacht hätten ${ }^{75}$. Doch: Nicht alles, worüber in einem Vertrag eine Regelung fehlt, stellt eine durch Auslegung zu füllende Vertragslücke dar ${ }^{76}$. In vielen Fällen soll nach dem Willen der Parteien vielmehr nur das Gesetz die Rechtsfolge bestimmen. Keinesfalls darf im Wege ergänzender Vertragsauslegung ein Ergebnis gefunden werden, das dem erkennbaren Willen der Vertragsparteien widerspricht ${ }^{77}$.

Nach Ansicht des Senats gehören bei der ergänzenden Vertragsauslegung die in dem Vertrag enthaltenen Angaben über dessen Zweck, aber auch die eigenen Angaben des Experten zu Inhalt und Umständen der Auftragserteilung „zum wesentlichen Auslegungsstoff"78. Die Weitergabe des Prüfberichts an Dritte „durch die Aufnahme in den Prospekt und die Verwendung des Prospekts bei derZeichnung von Aktien durch Anleger" sei in dem vorliegenden Fall „Grundlage des Auftrags“ gewesen ${ }^{79}$. In dem „Bericht über die Prüfung des Prospektes über Aktien“ vom 25.4.2007 - abgedruckt auf S.60-62 des Wertpapierprospekts - werde der Auftragsinhalt unter Bezugnahme auf das WpPG und die europäische ProspektVO beschrieben und abschließend festgestellt, dass „die

73 Zum Meinungsstand Medicus/Lorenz, SchuldR AT, 19. Aufl., 2010, S. 393 m. w. N.; Евке, JZ 1998, 991, 993.

74 BGH NZG 2014, 741, 741 (Rdn. 9).

75 Siehe nur BGH NJW 2002, 2310, 2311; BGH NJW-RR 2013, 494, 495 Rdn. 12.

76 So BGH NJW 1963, 2071, 2075.

77 Vgl. BGH NJW 1995, 1212, 1213; BGH NJW 2009, 1482, 1484 Rdn. 24.

78 BGH NZG 2014, 741, 742 (Rdn. 16).

79 BGH NZG 2014, 741, 742 (Rdn. 16). 
Gewinnprognosen und Gewinnschätzungen der Emittentin auf der angegebenen Grundlage ordnungsgemäß erstellt wurden und in Einklang mit den Rechnungslegungsstrategien der Gesellschaft stünden"80. Die Bekl. habe wissen müssen, dass „der nach $\ 3 \mathrm{WpPG}$ zu veröffentlichende Wertpapierprospekt und damit auch ihr Prüfbericht Anlegern im Vorfeld des Erwerbs der auszugebenden Namensaktien als Informationsgrundlage zur Verfügung gestellt werden würde“81. Die Berufung der Bekl. auf Nr.7 der (in dem Prospekt auf S. 72-73) abgedruckten AAB vom 1.1.2002 lässt der Senat - wie auch das OLG Düsseldorf ${ }^{82}$ - nicht gelten. Insbesondere Nr. 7 Abs. 1 Satz 1 der AAB ${ }^{83}$ erfasse nicht „einen Fall wie den vorliegenden, in dem sich der Prüfer gerade vertraglich verpflichtet, eine zur Veröffentlichung in einem Prospekt bestimmte Bewertung zu Gunsten zukünftiger Anleger abzugeben “84. Der Senat geht deshalb von einer „,individuellen Einbeziehung der Anleger in den Vertrag“ aus, „die die allgemeinen Regelungen in den $\mathrm{AAB}$ verdrängt" ${ }^{\text {"85. }}$.

Der Senat nimmt an, dass wegen Nr. 7 Abs. 1 Satz 1 AAB eine „Dritthaftung ... von vornherein ausgeschlossen “ wäre, da bezüglich der zum Zeitpunkt des Vertrages noch unbekannten Anleger natürlich keine, Einwilligung zu einer Weitergabe an einen bestimmten Dritten “" vorlag ${ }^{86}$. Doch: Warum soll sich aus dem Inhalt des Prüfungsauftrags vom 21.3.2007 in Verbindung mit Nr.7 Abs. 1 Satz 1 AAB nicht die Einwilligung zur Weitergabe des Prüfberichts an (potentielle) Wertpapiererwerber ergeben können, auch wenn diese bei Abschluss des Auftrags oder zu einem späteren Zeitpunkt (z. B. der vereinbarten Einbeziehung des Prüfberichts in den Wertpapierprospekt) zahlenmäßig oder namentlich noch nicht bekannt waren? „Einen“ muss ja nicht als Zahl verstanden werden, sondern könnte auch ein unbestimmter Artikel sein. Und unter „bestimmten Dritten“ könnte eine „überschaubare, klar abgegrenzte Personengruppe ${ }^{\text {"87 }}$ zu verstehen sein, in casu die (potentiellen) Wertpapiererwerber, für die der Prüfbericht der Bekl. durch die vereinbarte Einbeziehung in den Prospekt und die Verwendung des Prospekts bei der Zeichnung von Aktien durch Anleger bestimmt war ${ }^{88}$. So gesehen wäre die

80 BGH NZG 2014, 741, 742 (Rdn. 16).

81 BGH NZG 2014, 741, 742 (Rdn. 18).

82 OLG Düsseldorf BeckRS 2014, 17539.

83 Nr.7 Abs. 1 Satz 1 der AAB lautet: „Die Weitergabe beruflicher Äußerungen des Wirtschaftsprüfers (Berichte, Gutachten und dgl.) an einen Dritten bedarf der schriftlichen Zustimmung des Wirtschaftsprüfers, soweit sich nicht bereits aus dem Auftragsinhalt die Einwilligung zur Weitergabe an einen bestimmten Dritten ergibt“.

84 BGH NZG 2014, 741, 742 (Rdn. 19).

85 BGH NZG 2014, 741, 742 (Rdn. 19).

86 BGH NZG 2014, 741, 742 (Rdn. 19).

87 So der X. Zivilsenat des BGH in JZ 1998, 624, 627 zur Einbeziehung eines Darlehnsgebers in den Schutzbereich eines Vertrages.

88 So der III. Zivilsenat des BGH in WM 1995, 204, 205 (betr. einen Bausachverständigen). 
in dem Auftragsschreiben der Bekl. vom 21.3.2007 enthaltene Bemerkung, wonach sich ihre Haftung für die Durchführung der Prüfung auch gegenüber Dritten auf 4 Mio. Euro beschränke, nicht „überflüssig“89, sondern unterstriche ihren Willen, dass die Haftungsbegrenzung aus dem Grundvertrag (vgl. Nr. 9 Abs. 2 Satz 1 1. Halbsatz AAB) auch im Verhältnis zu Dritten Wirkung entfalten soll, wenn eine Haftung gegenüber einer anderen Person als dem Auftraggeber begründet sein sollte (vgl. Nr.9 Abs. 2 Satz 1 2. Halbsatz $\mathrm{AAB})^{90}$. Die Haftungsbeschränkung würde jedenfalls einer Existenz gefährdenden Dritthaftung des Prüfers vorbeugen.

\section{Versicherungslösung}

Die Frage nach der Höhe der Haftung der Bekl. gegenüber den Wertpapiererwerbern stellt sich auch, wenn man der Ansicht des Senats folgt und von einer „individuellen Einbeziehung der Anleger in den Vertrag“ ausgeht ${ }^{91}$. Ein Eingreifen der Haftungsbeschränkung aus dem Grundvertrag zwischen Emittent und Wirtschaftsprüfungsgesellschaft im Verhältnis zu Personen, die in den Schutzbereich des Vertrages einbezogen sind, ist trotz des auf den drittschützenden Vertrag entsprechend anzuwendenden $\$ 334$ BGB $^{92}$ in der Praxis keineswegs gesichert; bisweilen wird sogar angenommen, dass die Haftungsbeschränkung aus dem Grundvertrag im Verhältnis zu Dritten „konkludent“ abbedungen sei ${ }^{93}$. Eine uferlose Ausweitung der Haftung des Wirtschaftsprüfers aus einem drittschützenden Vertrag befürchtet der Senat dennoch nicht. Er verweist - wie das OLG Düsseldorf ${ }^{94}$ und das LG Duisburg95 vor ihm - insoweit auf die „Versicherungslösung“: „[D]as durch die zu zeichnende Kapitalsumme begrenzte Gesamtrisiko [der Wirtschaftsprüfungsgesellschaft] sei gegebenenfalls versicherbar und in die Vergütung einkalkulierbar gewesen "96. Die Berufshaftpflichtversicherung" wird so zum Instrument für die „Sozialisierung“ individueller Vermögensschäden der Ka-

89 BGH NZG 2014, 741, 742 (Rdn. 19).

90 Zur Einbeziehung der AAB in den Prüfungsvertrag siehe Eвke, ZVglRWiss 109 (2010), 397, 410-411.

91 BGH NZG 2014, 741, 742 (Rdn. 19).

92 Vgl. LG Frankfurt a. M. BB 1997, 1682, 1683.

93 Siehe dazu näher EвкE, aаO (Fn. 3), \323 HGB Rdn. 159 m. w. N.

94 OLG Düsseldorf BeckRS 2014, 17539.

95 LG Duisburg BeckRS 2014, 09272.

96 BGH NZG 2014, 741, 743 (Rdn. 20). Nach den Angaben des LG Duisburg betraf die Emission 3.750.000 Stück Aktien zu einem Ausgabepreis von je 3,60 Euro, sie hatte also ein Gesamtvolumen von 13,5 Mio. Euro: LG Duisburg BeckRS 2014, 09272.

97 Zur Berufshaftpflichtversicherung der Wirtschaftsprüfer siehe EвкE, aаO (Fn. 3), \323 HGB Rdn. 9-13. 
pitalanleger ${ }^{98}$. Ruinöse Folgen für den Prüfer sind nicht zu besorgen, solange eine entsprechende Versicherung am Markt erhältlich ist und die Kosten einer solchen Versicherung auf den Auftrag gebenden Emittenten überwälzt werden können ${ }^{99}$. Doch die Praxis sieht oft anders aus: Das Honorar für die Bestätigung von Gewinnprognosen in Prospekten ist meist begrenzt und dementsprechend die Bereitschaft des Mandaten, die Kosten einer Versicherung des die zu zeichnende Kapitalsumme abdeckenden Risikos zu übernehmen bzw. sich daran nennenswert zu beteiligen. Die Erfahrung mit der Zeichnung von Versicherungsschutz für die Erstelllung von Comfort Letters zeigt zudem, dass die Emittenten, aber auch die Versicherer bei höheren Emissionsvolumina in der Regel nur zur Zeichnung eines diese Volumina nur teilweise abdeckenden Versicherungsschutzes bereit sind.

\section{Kausalitätserwägungen}

Die in Fällen der vorliegenden Art oft schwierigen Kausalitätsfragen wurden in casu dadurch erleichtert, dass die Bekl. die Feststellungen des OLG Düsseldorf, dass sie ihre vertraglichen Pflichten schuldhaft verletzt und der Zedent seine Anlageentscheidung im Vertrauen auf die Richtigkeit des Prüfberichts getroffen hat ${ }^{100}$, in der Revision nicht angegriffen hat ${ }^{101}$. Gleichwohl bleibt die Frage, ob im Interesse der Bekl. Raum ist für mildernde Korrekturen an den Kausalitätserwägungen des OLG Düsseldorf. In Betracht kommt (außer der Adäquanztheorie ${ }^{102}$ ) die Schutzbereichslehre (enger formuliert: die Lehre vom Rechtswidrigkeitszusammenhang), wonach nur diejenigen Folgen beachtet werden sollen, die noch im Schutzbereich der von dem Prüfer verletzten Rechtsnorm oder Vertragspflicht liegen ${ }^{103}$. Letztlich wird hierdurch der Zweck des Vertrages für eine Einschränkung des zu leistenden Schadensersatzes nutzbar gemacht ${ }^{104}$. Darüber hinaus ist zu erwägen, ob der Zedent den Schaden nicht als Teil seines allgemeinen Lebensrisikos selbst tragen muss. Durch diese Korrektur wird aus dem schlichten (weil auf die Logik beschränkten) Kausalzusammenhang ein „wertender Zurechnungszusammenhang “105. Der Senat geht entgegen der Auffassung der Bekl. davon aus, dass

98 Zur Rolle der Dritthaftung und der Berufshaftpflichtversicherung als Instrument der Sozialisierung eingetretener Vermögensschäden siehe Евке, Die zivilrechtliche Verantwortlichkeit der wirtschaftsprüfenden, steuer- und rechtsberatenden Berufe im internationalen Vergleich, 1996, S. 36.

99 Евке, 79 Nw. U. L. Rev. 663, 682 (1984).

100 OLG Düsseldorf BeckRS 2014, 17539.

101 BGH NZG 2014, 741, 743 (Rdn. 23).

102 Siehe dazu statt vieler Medicus/Lorenz, aaO (Fn. 73), S. 306-307.

103 Medicus/Lorenz, aaO (Fn. 73), S. 307-309.

104 Vgl. Medicus/Petersen, Grundwissen zum Bürgerlichen Recht, 10. Aufl., 2014, S. 76.

105 Vgl. Medicus/Petersen, aaO (Fn. 104), S. 76. 
die Voraussetzungen für derartige mildernde Korrekturen in casu nicht vorliegen und es dem Schutzzweck der verletzten Vertragspflicht entspricht, die Haftung des Prüfers nicht lediglich auf „etwaige Schäden aus einer geringeren oder unterbliebenen Gewinnerwartung" zu beschränken, sondern auf den Verlust des für den Erwerb des Anteils aufgewandten Betrages ${ }^{106}$. Die Begründung des Senats ist nachvollziehbar und im Ergebnis nicht zu beanstanden.

\section{Verjäbrung}

Der Senat kommt der Kl. noch in einem weiteren Punkte entgegen: Nach Ansicht des Senats war im Zeitpunkt der Klageerhebung Anfang 2011 eine Verjährung des Schadensersatzanspruchs noch nicht eingetreten ${ }^{107}$. Wenn bei einem Schadensersatzanspruch der Haftung auslösende Fehler in einer fehlerhaften Rechtsanwendung des Prüfers liege, könne nicht die Kenntnis dieser Rechtsanwendung als solche ausreichen; vielmehr müsse der Geschädigte Kenntnis oder grobe Unkenntnis davon haben, dass die Rechtsanwendung fehlerhaft gewesen sei. Das sei aber nicht anzunehmen ${ }^{108}$. Natürlich kann man nicht bei jedem Wertpapiererwerber bilanzrechtliche Kenntnisse unterstellen und eigenständige rechtliche Prüfungen verlangen ${ }^{109}$. Man kann aber die Frage stellen, ob das Scheitern der Kapitalerhöhung und die nie erfolgte Übertragung der Anteile nicht Anlass genug gewesen sein könnten, den Prüfbericht der Wirtschaftsprüfungsgesellschaft in dem Prospekt fachkundig überprüfen zu lassen. Der Senat verneint diese Frage, weist aber zugleich darauf hin, dass bei einer gegenteiligen Ansicht die Verjährungsfrage nicht anders zu entscheiden gewesen wäre, weil die Kapitalerhöhung nach dem Vortrag der Bekl. selbst erst am 1.2.2008 endgültig gescheitert sei und die Frage einer anschließenden Überprüfung des Prüfberichts für die Verjährung mithin nicht entscheidungserheblich war.

\section{Ausblick}

Das vorstehende Urteil zeigt, dass Wirtschaftsprüfer, aber auch andere Experten im Interesse der Kapitalanleger große Sorgfalt walten lassen müssen, wenn sie Beiträge oder Materialien zu Wertpapierprospekten beisteuern oder auf andere Weise an der Erstellung eines solchen Prospekts mitwirken, ohne die Verantwortung für die Richtigkeit des Prospekts zu übernehmen. Es bleibt zu hoffen, dass der BGH in derartigen Fällen vor allem bei leichter Fahrlässigkeit 
des Experten den Gesichtspunkt der praktischen Konkordanz im Auge behalten und bezüglich der Voraussetzungen einer zivilrechtlichen Haftung (z. B. nach den Grundsätzen des Vertrages mit Schutzwirkung für Dritte oder der allgemeinen zivilrechtlichen Prospekthaftung) mit Augenmaß vorgehen wird. Gleiches gilt für die vielen anderen Aufgaben, die Wirtschaftsprüfern nach $\$ 2$ WPO zugewiesen sind und deren (Schlecht-)Erfüllung Gegenstand von Schadensersatzklagen sein kann, die auf Grund der Geschäftsverteilung des $\mathrm{BGH}^{110}$ höchstrichterlich von sechs verschiedenen Senaten entschieden werden.

110 Die Verteilung der richterlichen Aufgaben auf die Senate des BGH und die Zusammensetzung der einzelnen Senate werden durch den Geschäftsverteilungsplan bestimmt, den das Präsidium des BGH vor Beginn eines jeden Geschäftsjahres für dessen Dauer beschließt. Der vollständige Geschäftsverteilungsplan wird einmal im Jahr in einer Beilage zum Bundesanzeiger sowie auf der Internetseite des BGH veröffentlicht. 